\title{
Exercise, Health, and Falls Risks among Older African American Women
}

\author{
Maria Kosma ${ }^{*}$, Jan M. Hondzinski², David R. Buchanan ${ }^{3}$ \\ ${ }^{1}$ School of Kinesiology, Louisiana State University, 331 Peabody Hall, Baton Rouge, Louisiana 70803, Baton Rouge, USA \\ ${ }^{2}$ School of Kinesiology, Louisiana State University, 112 Huey P. Long Field House, Baton Rouge, Louisiana 70803, Baton Rouge, USA \\ ${ }^{3}$ School of Public Health \& Health Sciences, University of Massachusetts, 306 Arnold House, Amherst 01003, Amherst, MA
}

Corresponding Author: Maria Kosma, E-mail: mkosma@1su.edu

\section{ARTICLE INFO \\ Article history \\ Received: Apr 27, 2017 \\ Accepted: June 30, 2017 \\ Published: July 31, 2017 \\ Volume: 5 Issue: 3}

Conflicts of interest: None

Funding: None

\begin{abstract}
Background of Study: Although exercise has many benefits, older African American (AA) women are less active than older Caucasian women and older AA men. Balance and musclestrengthening activities are typically recommended for decreased falls, whereas the role of aerobic training alone on falls prevention is controversial. Objective: This was a mixed methods phronetic (pragmatic) study - without an intervention - including quantitative data (falls risks) and qualitative data on exercise behavior and its importance to health and falls prevention; therefore, the studied phenomenon was thoroughly and pragmatically investigated. The first purpose of the study was to examine differences in falls risks based on exercise type (aerobics vs. combination of aerobics, muscle training, and balance activities) and exercise level (active people vs. somewhat active people). Secondly, participants' exercise values were examined in relation to their health, falls-risk prevention, exercise behavior, and falls risks. Method: Interviews and falls risk assessments were conducted among 12 older AA women in an inner-city community center. Results: ANCOVA and ANOVA showed that the aerobics group performed better in Dynamic Gait Index (DGI) and Timed Up and Go than the combination group $(\mathrm{d}=0.85,-0.97)$; the latter surpassed the former in Functional Reach $(\mathrm{d}=2.27)$. The active group (met the 150 minutes/week exercise recommendation) performed better in DGI and SixMinute Walk than the somewhat active group $(d=0.62 ., 50)$; the latter outperformed the former in balance-eyes open $(d=-0.52)$. Emerging themes about lifestyle values included: a) reasons for health conditions and staying healthy and b) falls prevention. Conclusion: Exercise programs for fall risk reduction should include not only muscle strengthening and balance activities, but also aerobic exercises. Meeting minimum exercise recommendations is key to falls risk reduction. Beyond healthy diet, the role of exercise on the prevention of health conditions needs to be emphasized.
\end{abstract}

Key words: Exercise, Falls Risks, Health, Older African American Women, Phronetic Mixed-Methods Research

\section{INTRODUCTION}

Although exercise participation has multiple benefits among older adults, including decreased risk of diabetes, lowered cholesterol levels, and decreased falls risks (Panel on Prevention of Falls in Older Persons, American Geriatrics Society and British Geriatrics Society [AGS/BGS], 2011; Sadeghi et al., 2017; Shumway-Cook, Gruber, Baldwin, \& Liao, 1997; Sigal, Wasserman, \& Castaneda-Sceppa, 2004), only $36.5 \%$ of older adults meet the minimum aerobic recommendations - 150 minutes/week - compared with $46.2 \%$ of adults aged 45-64 years old (US Department of Health and Human Services [USDHHS], 2016a). Racial disparities in exercise participation and health also exist with older African American (AA) women being less active than older
Caucasian women and older AA men (Federal Interagency Forum on Aging-Related Statistics, 2016). Moreover, African Americans have higher rates of diabetes, hypertension, and deaths from coronary heart disease and stroke than Caucasians (USDHHS, 2016b, 2016c), thus could potentially reduce these rates with greater exercise participation.

About $33 \%$ of older adults fall each year and falling can lead to serious injury or death (Inouye, Brown, \& Tinetti, 2009). Limited mobility, muscle strength, endurance, and balance are considered intrinsic falls risk factors that can be reduced by exercise programs (Ambrose, Paul, \& Hausdorff, 2013). Although a combination of resistance and balance training and endurance exercises has been used for the prevention of falls, the general exercise recom- 
mendation for falls prevention is participation in balance and muscle-strengthening activities (Panel on Prevention of Falls in Older Persons, AGS/BGS, 2011; Sherrington, Tiedemann, Fairhall, Close, \& Lord, 2011). The role of aerobic exercise alone in falls prevention is controversial. Endurance exercises like walking are either considered an unnecessary addition in exercise programs for falls prevention (Sherrington et al., 2011) or a secondary modality following balance and muscle training activities (Karlsson, Vonschewelov, Karlsson, Coster, \& Rosengen, 2013). However, given the link between endurance training and falls prevention - via either improved physical function or prevention of diabetes and management of neuromuscular disorders (Crews, Yalla, Fleischer, \& Wu, 2013; Pieterse, Luttikhold, de Laat, Bloem, van Engelen, \& Munneke, 2006), some researchers view aerobic training as a necessity in exercise falls-prevention programs (Paterson \& Warburton, 2010). Although qualitative studies on benefits and barriers of lifestyle behaviors - such as exercise and diet - among older adults exist (Franko et al., 2015; Haynes-Maslow et al., 2013), to our knowledge there is no qualitative literature on the importance of exercise on falls prevention and attributions of health conditions, especially among older AA women. Examining attributions to health problems and ways to deal with them can enlighten understanding about value systems regarding healthy lifestyle behaviors, such as exercise.

The voice of community members becomes as important as the expertise of investigators to better understand people's exercise values (Kosma, Buchanan, \& Hondzinski, 2015). Human action, such as exercising and eating healthy, can be understood via phronesis or practical wisdom, which is influenced by context-dependent and varied life experiences within one's culture and society (Aristotle, 350 B.C.E./1962; Kosma et al., 2015): what do I think about my health? Is exercise important and why? People decide to participate in behaviors that they find valuable based on external influences - history and cultural upbringing - and their own assessments regarding what is meaningful in life (Wolf, 2015). They may ask: is it wise to be inactive and eat out often like most of my friends or shall I start exploring different lifestyles from other cultures? What is the meaning of health in my life? Phronetic (pragmatic) research is concerned with understanding meaningful human behavior (e.g. exercise). Although phronetic research is typically qualitative in nature, mixed-methods designs incorporating qualitative and quantitative data are also part of phronetic research paradigms in order to thoroughly and pragmatically explain a phenomenon in relation to human action (Flyvbjerg, 2001, 2004). Flyvbjerg (2004) highlights the fluidity of research methodology in phronetic designs, which are not supposed to be method-driven but pragmatic in nature in order to address an imminent issue. In his very own Aalborg project, Flyvberg (2001) implemented both quantitative (e.g., survey data) and qualitative techniques (e.g., interviews with the chief of city planning) to alter the downtown center of the city of Aalborg in North Denmark, from a highly polluted and unsafe place because of heavy traffic to a green place friendly to pedestrians, bikers, and tourists (Flyvbjerg, 2001; Kosma et al., 2015). Given the equivocal role of aerobic exercise on falls prevention and the potential influence of lifestyle values on health, the purpose of this phronetic mixed methods study was to integrate quantitative data (falls risks) with qualitatively derived data (exercise type, level, and its importance in relation to falls risk prevention and health) among older AA women in order to explore the following. First, differences in falls risks (quantitative data) based on exercise type (aerobics vs. combination of aerobics, muscle training, and balance activities) and exercise level (active people - met the 150 minutes/week guidelines - vs. somewhat active people) were investigated. Second, participants' exercise values were examined in relation to their health, falls-risk prevention, exercise behavior (type and level), and falls risks (quantitative data).

\section{METHODS}

\section{Design and Procedures}

This mixed methods phronetic study involved quantitative and qualitative data collected in an inner-city community center. This was not an experimental design (i.e., without an intervention); the quantitative data derived from objective falls-risk/functional measures and the qualitative data reflected exercise behavior (type and level) and its importance to health and falls prevention. After learning about the study's purpose and procedures, participants signed the corresponding consent form approved by the University's Institutional Review Board (\#3558). Participants were screened for their ability to perform functional tests and cognitive function via the revised seven-item Physical Activity Readiness-Questionnaire (PAR-Q; Canadian Society for Exercise Physiology, 1994) and the Mini-Mental State Examination (MMSE; Folstein, Folstein, \& McHugh, 1975), respectively. In this study, all qualified participants had acceptable cognitive function: MMSE range of scores $=25-30, \mathrm{M}=28 \pm 1.76$, which were greater than the 24 cutoff score for evidence of cognitive decline (Folstein et al., 1975). Based on the PAR-Q, affirmative (Yes) responses to health issues related to heart, musculoskeletal, and neurological problems, which would normally disqualify seven study participants from the falls risk measures, were overridden in cases of physician's recommendation of exercise participation (Canadian Society for Exercise Physiology, 1994). Initially, the director of the community center recruited 21 study participants. Of those individuals, 12 older AA women qualified to be in the study. Reasons for non-participation included: one disqualification based on the PAR-Q score and six cancelations because of harsh weather or a mix-up on dates. Rescheduling interviews was difficult due to various time constraints. Two additional women were excluded from the study because they were below 60 years of age to qualify for older adult falls-risk norms (Bohannon, 2006). Based on the dominant positivism methodological paradigm, the number of study participants for the quantitative data may be considered small for generalizability purposes. However, in phronetic (pragmatic) research generalizability is not the main focus. Additionally, 
phronetic data (quantitative or qualitative) can be generalizable even with one case dependent on the importance of the investigation, the paradigmatic nature of the case(s), and quality of results (Flyvbjerg, 2001). It is also important to highlight the challenges in recruitment of underserved populations (e.g. older AA adults in inner cities) in exercise promotion studies (Kosma, Cardinal, \& McCubbin, 2004). In this (phronetic) study, the participants were chosen because they reflected educationally and economically diverse older AA women of varied exercise levels, health perspectives, and socio-cultural backgrounds. Therefore, the sample size for both the quantitative and qualitative piece of this phronetic approach is considered conceptually and methodologically sufficient.

\section{Qualitative Data Collection}

Following the screening of the participants, individual interviews were conducted in a quiet room and audio-taped by the first author. According to phronetic qualitative research (Flyvbjerg, 2001; Kafle, 2011), the interview process was dialogical in nature in order to build trust for in-depth discussions with the participants. Although an interview guide was used (Table 1), the order and content of the questions varied from case to case. Depending on shared experiences, the emphasis of the discussion vacillated between social aspects of exercising and fitness improvement (see Kosma, Buchanan, \& Hondzinski, in press). Interview questions included typical current and recent past - within last year - exercise behavior, health conditions, reasons for health problems, and importance of exercise (Table 1). The beneficial exercise effects on falls risk and exercise last at least one year post-intervention among older women, thus the chosen one-year period for recent past exercise behavior (Liu-Ambrose, Khan, Eng, Gillies, Lord, \& McKay, 2005). Additional interview questions were asked of 14 women which included the 12 participants and the results are reported elsewhere (Kosma et al., in press). At the end of the interviews, participants reported age, ethnicity, education, income, and number of household members. The length of each individual interview varied between 23 minutes and 47 minutes. The interview guide was initially drafted by the first author and reviewed by the two co-authors and the director of the community center for revisions and recommendations. The revised questions were then pilot-tested with two participants, one of whom was included in the study while the other was excluded because of her younger age.

Table 1. Interview guide - Questions

What does your typical day look like? What are your daily
activities?
What do you think about your physical health? Do you have
any health conditions?
Why do you think you have those conditions?
In what types of physical activities do you currently participate?
What are your current physical activity experiences?
What are your past experiences with physical activity?
Why are you physically active?

\section{Quantitative Measures}

Following the interviews, falls-risk measures included Timed Up and Go (TUG) (Podsiadlo \& Richardson, 1991), dynamic gait index (DGI) (Shumway-Cook, Baldwin, Polissar, \& Gruber, 1997), functional reach (FR) (Duncan, Studenski, Chandler, \& Prescott, 1992), six-minute walk test (6MWT) (Enright, 2003; Steffen, Hacker, \& Mollinger, 2002), and standing balance (sway) with eyes open (sway-EO) and eyes closed (sway-EC) (Melzer, Benjuya, \& Kaplanski, 2004; Moghadam et al., 2011). Multiple measures of falls risk can more accurately depict physical function (Thapa, Gideon, Brockman, Fought, \& Ray, 1996). These data are unique to this paper and they were not included in our previous qualitative study (Kosma et al., in press).

The TUG measure (in seconds), is the time taken by an individual to stand up from a standard arm chair, walk a distance of 3 meters, turn, walk back to the chair, and sit down. Time is compared to norms to identify if the person is at high, medium, or low risk for falls: low falls risk < $14 \mathrm{sec}$; high falls risk $>14 \mathrm{sec}$ with excellent test-retest reliability of intraclass correlation coefficient $=0.99$ (Podsiadlo \& Richardson, 1991). TUG also correlates well with balance, gait speed, and functional capacity supporting its construct validity (Podsiadlo \& Richardson, 1991; Steffen et al., 2002). In the FR, the participant stood next to a wall with one foot next to the other and the dominant arm flexed perpendicularly, in a position horizontal to the frontal plane (straight forward from the shoulder joint). From this location, the participant was asked to reach as far forward as possible without losing their balance. After a few practice trials, distance in inches between start and final hand position is recorded for three trials and averaged to provide a test-retest reliability $=0.92$ (Duncan et al., 1992). Fallers could be distinguished from non-recurrent fallers based on FR, establishing its predictive validity. Cutoff scores for falls risks are as follows: low falls risk $>10$ inches; moderate risk $=6$-10 inches; high risk $<6$ inches (Duncan et., 1992). The DGI uses eight test items including level walking with and without horizontal or vertical head turns, speeding up or slowing down, using a pivot turn, as well as stepping over or around objects and managing stairs, and the score corresponds to the person's ability to maintain normal gait pace within a 15 inch pathway without staggering or stumbling during walking (Shumway-Cook et al., 1997). The measure has exhibited excellent test-retest reliability exceeding 0.96 and performances in the current study were video-taped and reviewed by multiple evaluators to ensure consistent scoring. Older adults who adhered to a balance and mobility program had better scores in DGI than the partially adherent exercise group to support its construct validity (Shumway-Cook et al., 1997). The range for low falls risk is 19-24 while scores below 19 are predicative of falls (Shumway-Cook, Baldwin, Polissar, \& Gruber, 1997). The 6MWT measures the distance in feet that a person can walk in 6 minutes, which originally assessed the functional capacity of people with at least moderately severe impairment (Enright, 2003). The age-based cutoff norms for older adults living independently and self-reliant in activities of daily living are: $60-69$ years $=1165-2375$ feet; 
70-79 years $=1056-2043$ feet; 80-89 years $=731-1849$ feet with test-retest reliability of 0.95 (Steffen et al., 2002). Older age and health conditions such as chronic obstructive pulmonary disease associate with shorter distance in the 6MWT supporting its construct validity (Enright, 2003; Steffen et al., 2002). For standing sway (balance), center of pressure (CoP) data were collected while participants stood still on a force plate with their feet close together for 30 seconds, first with eyes open and then with eyes closed. CoP is used to calculate different measurements of sway that are compared to norms to assess good or poor balance. The mean velocity of the CoP used to estimate falls risks based on mean of non-fallers plus 3 SD are as follows: sway-EO faller $>2.2 \mathrm{~cm} / \mathrm{s}$; sway-EC faller $>2.9 \mathrm{~cm} / \mathrm{s}$ (Melzer et al., 2004). Test-retest reliability for older adults is acceptable: .89 for eyes open and .70 for eyes closed (Moghadam et al., 2011). Fallers tend to score higher (worse) in sway than non-fallers supporting its construct validity (Melzer et al., 2004).

\section{Qualitative Analysis}

Audiotapes of the interviews were transcribed verbatim. The interviewer double-checked transcription accuracy. The thematic analysis and its rigor were identified through analytical steps of phronetic qualitative research (Flyvbjerg, 2001; Kafle, 2011): a) Data were entered in NVivo 10 and the transcripts were read and reread several times by the first and third authors to become familiar with the participants' stories; b) the same authors independently coded the data in a systematic fashion and collated the data pertinent to each code; c) codes were collated into potential themes and sub-themes; d) developed themes and sub-themes were reviewed, named, and refined by all study authors in relation to the coded data and entire story; and e) compelling quotes relevant to each individual story and the entire data set were extracted in a process also known as the hermeneutic circle (Kafle, 2011). In phronetic qualitative research, reaching theoretical saturation is not relevant or of interest. Instead, key indicators in testing the rigor of this qualitative investigation involved the emphasis on values, the involvement of participants and the center director in developing the interview guide, the dialogical nature of the interviews, and the in-depth examination and report of the participants' stories via reflective reading and rigorous interpretation by all researchers (Flyvbjerg, 2001; Kafle, 2011; Kosma et al., 2015). Conflicts in assigning specific codes were resolved through consensus discussions.

\section{Quantitative Analysis}

The Statistical Package for the Social Sciences (SPSS, version 23) was used for the quantitative analyses. Descriptive statistics (Means and $S D$ ) and Pearson Product Moment correlations of the falls risk measures were examined. After controlling for age and health condition type (diabetes and/or neuromuscular condition vs. no diabetes or neuromuscular disorder) (Crews et al., 2013; Pieterse et al., 2006), univariate analyses of covariance (ANCOVA) and ANOVA for FR were used to examine differences in falls risks based on exercise type (aerobics vs combination) and exercise level in minutes (active vs. somewhat active). Due to the small sample size for the omnibus $F$ tests, Cohen's $d$ effect size and variance explained $\left(\eta^{2}\right)$ were computed with low, moderate, and large values as follows: $d=0.2,0.5,0.8 ; \eta^{2}=1 \%, 6 \%, 14 \%$ (Cohen, 1988). The use of effect sizes is recommended in ANOVA and ANCOVA with small samples (Cortina \& Nouri, 2000; Lakens, 2013). To avoid overestimation of SPSS partial $\eta^{2}$ values in ANCOVA, all actual $\eta^{2}$ values were hand-calculated (Levine \& Hullett, 2002).

\section{RESULTS}

\section{Participant Characteristics, Falls Risk Correlations, and Individual Falls Risks}

The average age of the 12 older AA women was 70 years old $( \pm 8.28$; age range $=61-86)$. The participants were fairly well-educated and their income levels varied from $\$ 7,000$ to $\$ 125,000$ (Table, 2). Most of the women lived alone $(n=7)$, while there were households with two people $(n=2)$ and three people $(n=3)$. Based on the interviews, reported health conditions included high blood pressure/hypertension $(n=5)$ and high cholesterol $(n=7)$, arthritis $(n=4)$, diabetes $(n=3)$, asthma $(n=2)$, heart condition $(n=1)$, dystonia $(n=1)$, multiple sclerosis $(n=1)$, and peripheral neuropathy $(n=1)$. Overall, falls-risk correlations were in the expected direction (Table 3). The unexpected direction of two small correlations - FR-TUG, FR-DGI - likely result from the high variance in FR (Kline, 2015). In general, participants exhibited low falls risks and seven of them displayed increased risk in mainly one measure (see Table 4 - participant names are pseudonyms).

\section{Exercise Groups - Qualitatively Derived}

According to participants' reported current and recent past exercise behavior, half of the women met the minimum 150 minutes/week exercise guidelines (active) while the other half did not meet the guidelines, and thus they were

Table 2. Demographic profile

\begin{tabular}{lc}
\hline Variable & $\mathbf{N}(\mathbf{\%})$ \\
\hline Race & $12(100)$ \\
$\quad$ African American & \\
Education & $2(16.7)$ \\
Less than $9^{\text {th }}$ grade & $1(8.3)$ \\
High school/GED & $3(25)$ \\
Some college, no degree & $3(25)$ \\
College graduate & $3(25)$ \\
Graduate degree & \\
Income & $4(33.3)$ \\
$\leq \$ 19,000 /$ year/house member & $6(50)$ \\
$\geq \$ 21,000 /$ year/house member & $2(16.7)$ \\
Missing &
\end{tabular}


Table 3. Pearson correlations, Means, and SD of Falls risk variables

\begin{tabular}{|c|c|c|c|c|c|c|c|c|}
\hline & 1 & 2 & 3 & 4 & 5 & 6 & $\mathbf{M}^{\mathbf{a}}$ & SD \\
\hline 1. TUG (seconds) & - & -0.43 & 0.19 & $-0.69 *$ & 0.47 & 0.40 & 10.48 & 2.77 \\
\hline 2. DGI & & - & -0.30 & 0.43 & -0.10 & -0.33 & 22.08 & 2.43 \\
\hline 3. FR (inches) & & & - & 0.39 & 0.07 & -0.05 & 13.88 & 3.5 \\
\hline 4. 6MWT (feet) & & & & - & -0.19 & -0.35 & 1439 & 273 \\
\hline 5. Sway-EO $(\mathrm{cm} / \mathrm{s})$ & & & & & - & $0.90 * *$ & 1.55 & 0.65 \\
\hline 6. Sway-EC $(\mathrm{cm} / \mathrm{s})$ & & & & & & - & 1.79 & 0.63 \\
\hline
\end{tabular}

** $\mathrm{p} \leq 0.01 . * \mathrm{p}<0.05$., ${ }^{\mathrm{R}}$ Range of scores: TUG (Timed Up and Go): 7.49 - 16.22; DGI (Dynamic Gait Index): 15 - 24; FR (Functional

Reach): 7 - 17; 6MWT (Six-Minute Walk Test): 1036 - 1808; Sway-EO (eyes open): .88 - 2.8; Sway-EC (eyes closed): .99 - 2.78

Table 4. Individual falls risk for identified measures

\begin{tabular}{ll}
\hline Name & Increased falls risk \\
\hline Andrea & \\
Cailyn & Functional reach \\
Delilah & $\begin{array}{l}\text { Dynamic gait index } \\
\text { Dynamic gait index } \\
\text { Elena }\end{array}$ \\
Sway - eyes open \\
Edith & \\
Janna & \\
Margaret & \\
Anny & Dynamic gait index \\
Walda & Dynamic gait index \\
Queran & Dynamic gait index \\
Rena & Functional reach \\
Sondra & Dynamic gait index \\
& Sway - eyes open \\
\hline
\end{tabular}

considered somewhat active. Additionally, three women performed only aerobic exercises while nine women performed a combination of aerobics, muscle strengthening, balance, and stretching activities (Table 5 - participant names are pseudonyms). Exercise classes involved either mainly stretching and strengthening activities (Janna and Walda) or a combination of strength training (using dumbbells), balance (with rope activities), and aerobic exercises (dancing and walking) (Queran, Elena, and Sondra). Although intensity levels were not systematically assessed, some participants reinforced the importance to exert effort when exercising, including perspiring, walking fast, and increasing heart rate. These results are reported in detail in the qualitative study (Kosma et al., in press).

\section{Differences in Falls Risks by Exercise Type}

After controlling for the covariates - age for DGI, 6MWT, and TUG; health condition type for sway-EO/EC - ANCOVA and ANOVA for FR revealed large effect sizes for DGI $(d=0.85)$, TUG $(d=-0.97)$, and FR $(d=2.27)$. The aerobics group $(n=3)$ performed better in DGI and TUG than the combination group $(n=9)$, while the latter exhibited higher flexibility and stability (FR) than the former (Table 6).

\section{Differences in Falls Risks by Exercise Minutes}

After controlling for age and health condition type, ANCOVA and ANOVA for FR identified medium effect sizes for DGI $(\mathrm{d}=0.62)$, 6MWT $(d=0.50)$, and sway-EO $(d=-0.52)$. The active group $(n=6)$ performed better in DGI and 6MWT than the somewhat active group $(n=6)$, whereas the latter performed better in sway-EO than the former (Table 7).

\section{Emerging Themes - Qualitative Data}

Two key themes emerged from the qualitative analysis: 1) reasons for health conditions and staying healthy and 2) falls prevention. Although the first part of theme 1 (reasons for health conditions) was predetermined (see Table 1), the second part of the $1^{\text {st }}$ theme (staying healthy), the $2^{\text {nd }}$ theme, and all sub-themes emerged from the analysis. Ways to manage health problems and the concept of falls prevention were not mentioned by the interviewer. Representative quotes are presented to comprehensively illustrate each theme. For the purposes of this document, participants' names have been replaced with pseudonyms. Overall, these qualitative data are unique to the current study and they were chosen because of their links to the quantitative data (falls risks) - examining relations among falls risks, exercise behavior, and exercise importance for health and falls prevention. The only similarities to our previous qualitative paper (Kosma et al., in press) involve: a) Queran's and Delilah's comments on "bone and joint health" and b) Sondra's comments on "independence;" however, the use of these comments vary between manuscripts.

\section{Theme 1: Reasons for health conditions and staying healthy}

Hereditary - family reasons

Seven participants mentioned that family or hereditary reasons caused most of their health problems, such as diabetes, high blood pressure and cholesterol levels. Although Walda did not know the causes of her arthritis and peripheral neuropathy, she indicated that high blood pressure and diabetes run in her family: "I did not have high blood pressure until I reached fifties, but most of my family had it much younger." 
Table 5. Exercise type - current and recent past* - and groupings

\begin{tabular}{|c|c|c|c|c|}
\hline Name & Aerobics & $\begin{array}{l}\text { Strength, balance, } \\
\text { stretching }\end{array}$ & Combination & Groupings \\
\hline Andrea & Walking, biking (current) & & $\begin{array}{l}\text { Gardening (summer and } \\
\text { spring; current) }\end{array}$ & $\begin{array}{l}\text { Somewhat active } \\
\text { combination }\end{array}$ \\
\hline Cailyn & Walking (recent past) & & & $\begin{array}{l}\text { Somewhat active } \\
\text { aerobics }\end{array}$ \\
\hline Delilah & $\begin{array}{l}\text { Walking when weather is } \\
\text { nice (current) }\end{array}$ & Yoga (current) & & $\begin{array}{l}\text { Somewhat active } \\
\text { combination }\end{array}$ \\
\hline Elena & $\begin{array}{l}\text { Walking (current and recent past) } \\
\text { Dancing (current) }\end{array}$ & Exercises at home (current) & $\begin{array}{l}\text { Exercise class (current) } \\
\text { Yard work (current) }\end{array}$ & $\begin{array}{l}\text { Active } \\
\text { combination }\end{array}$ \\
\hline Edith & & & Gardening (current) & $\begin{array}{l}\text { Somewhat active } \\
\text { combination }\end{array}$ \\
\hline Janna & Walking (most of her life) & Exercise class (recent past) & & $\begin{array}{l}\text { Active } \\
\text { combination }\end{array}$ \\
\hline Margaret & Walking (recent past) & & & Active aerobics \\
\hline Anny & $\begin{array}{l}\text { Walking (current and recent past) } \\
\text { Treadmill (recent past) }\end{array}$ & $\begin{array}{l}\text { Abdominal exercises (current } \\
\text { and recent past) }\end{array}$ & $\begin{array}{l}\text { Cleaning buildings (current } \\
\text { and recent past) }\end{array}$ & $\begin{array}{l}\text { Active } \\
\text { combination }\end{array}$ \\
\hline Walda & Walking (current) & Exercise class (current) & & $\begin{array}{l}\text { Somewhat active } \\
\text { combination }\end{array}$ \\
\hline Queran & $\begin{array}{l}\text { Extra walking while } \\
\text { shopping (current) }\end{array}$ & At home (current) & $\begin{array}{l}\text { Exercise class (current and } \\
\text { recent past) }\end{array}$ & $\begin{array}{l}\text { Somewhat active } \\
\text { combination }\end{array}$ \\
\hline Rena & $\begin{array}{l}\text { Walking and biking (current for } \\
6-7 \text { years) }\end{array}$ & & & Active aerobics \\
\hline Sondra & & & $\begin{array}{l}\text { Exercise class and exercises at } \\
\text { home (current and recent past) }\end{array}$ & $\begin{array}{l}\text { Active } \\
\text { combination }\end{array}$ \\
\hline
\end{tabular}

*Recent past=within one year prior to data collection

\section{Lifestyle: diet and (in)activity}

Seven women attributed some of their health problems to unhealthy diet. Two women managed to decrease their excess weight and cholesterol levels by following healthier recent food choices than their past diet. For example, Anny believes that she now manages to keep her weight down due to her healthier diet:

"...well, my diet is very different now because I stay away from battered foods, no fried foods, no fast foods, and sweets... I was eating fast food and fried food and gaining weight... so I had to cut that out completely."

Rena managed to bring her cholesterol down "from 360 to 92 " due to healthy diet (e.g. fresh vegetables and fish). Following her doctor's recommendation, she started walking because she thinks walking "helps to lower it [high cholesterol] too." In addition to family reasons, only one individual, Margaret, attributed her arthritis to also inactivity: “...I think when you exercise, you keep your body fit and you uh... so another reason could be kind of... inactivity."

\section{Old age}

Two individuals attributed their arthritis to the normal process of aging, although this did not worry them. Edith downplayed her arthritis as not a real ailment but something that comes with age:
“It's like an age thing. It's not like an ailment. It's nothing you know that keeps you from doing things. It's just the aches and pains of old age. It's arthritis, rheumatoid arthritis... Just something that you get with old age I guess."

Stressing from discrimination and healing health problems

Queran was diagnosed with high blood pressure and cholesterol level when she was 50 years old because of high stress from unequal pay at work and perceived racial discrimination:

“... when I started they didn't want to pay us so they named us and when I say us- the black people. That is when I had... high blood pressure they say, high cholesterol... my dad did - he died early at 62. He had high blood pressure and he had a stroke - he was a civil rights worker; he and my mom both. We grew up civil rights workers and it was quite a bit of stress."

Astonishingly, she managed to treat her aforementioned health conditions, including fibromyalgia, via exercise and healthy diet:

"I think I healed that [fibromyalgia] myself you know with my eating and exercises... I decided that my food was going to be my medicine and my medicine was going to be my food." 
Table 6. Differences in falls risks by exercise type

\begin{tabular}{|c|c|c|c|c|c|c|}
\hline \multicolumn{7}{|c|}{ Exercise type } \\
\hline Variable & Aerobics $\mathbf{n}=\mathbf{3}$ & Combination $\mathbf{n}=9$ & $F * 1,9$ & $\mathbf{p}$ & $\eta^{2}$ variance $(\%)$ & d Effect size \\
\hline \multicolumn{7}{|l|}{ DGI } \\
\hline Mean & 23.33 & 21.67 & 0.37 & 0.56 & 2.5 & $0.85 * *$ \\
\hline SD & 0.58 & 2.7 & & & & \\
\hline \multicolumn{7}{|c|}{ 6MWT (feet) } \\
\hline Mean & 1371 & 1462 & 1.81 & 0.21 & 10 & 0.28 \\
\hline SD & 396 & 246 & & & & \\
\hline \multicolumn{7}{|c|}{ TUG (seconds) } \\
\hline Mean & 8.75 & 11.06 & 0.81 & 0.39 & 3.5 & $-0.97 * *$ \\
\hline SD & 1.71 & 2.89 & & & & \\
\hline \multicolumn{7}{|c|}{ FR (inches) } \\
\hline Mean & 9.33 & 15.39 & 15.85 & $<0.01$ & 61 & $2.27 * *$ \\
\hline $\mathrm{SD}$ & 3.21 & 1.98 & & & & \\
\hline \multicolumn{7}{|c|}{ Sway-EO (cm/s) } \\
\hline Mean & 1.38 & 1.6 & 0.47 & 0.51 & 2.4 & -0.33 \\
\hline SD & 0.64 & 0.68 & & & & \\
\hline \multicolumn{7}{|c|}{ Sway-EC (cm/s) } \\
\hline Mean & 1.78 & 1.79 & 0.00 & 0.99 & 0 & -0.01 \\
\hline SD & 0.73 & 0.64 & & & & \\
\hline
\end{tabular}

DGI: Dynamic gait index; 6MWT: Six-Minute walk test; TUG: Timed up and go; FR: Functional reach; EO: Eyes open; EC: Eyes closed, ${ }^{*} F$ degrees of freedom for FR: 1,10-ANOVA, **Large effect sizes for DGI, TUG, and FR

\section{Theme 2: Falls Prevention}

Bone and joint health

Six women acknowledged the importance of exercise for bone and joint health. Delilah said: "because I read a lot and I always read that as you age, your bones become brittle, things slow down. So if you remain active, you live longer. You are in better health." Queran mentioned that her "Walking Tall" class was important because "... when we get old our bones are brittle so we are trying to keep from falling." Anny highlighted the importance of exercise for joint health: “...keep moving so my joints won't get stiff."

\section{Be in shape (fit) and independent}

Four women exemplified the importance of exercise for increased body shape or functioning and independence. Margaret valued exercise to keep her body in the best shape possible. Elena and Sondra appreciated activity to maintain their functioning and independence. Sondra stated: “...I like to be able to do and not depend on anyone else."

\section{DISCUSSION}

The purpose of this phronetic (pragmatic) mixed methods study was to integrate quantitative data with qualitative data among older AA women of diverse education and income levels in order to explore the following. First, differences in falls risks (quantitative data) based on exercise type and level (qualitatively derived data) were investigated. Second, participants' exercise values were examined in relation to their health, falls-risk prevention, exercise behavior (type and level), and falls risks (quantitative data).

Exercise type did influence falls-risk measures. The aerobics group performed better in DGI and TUG than the combination group to support the importance of aerobics on mobility related falls risk. Older women who received only balance and muscle-strengthening training did not improve TUG scores (Patil, Uusi-Rasi, Tokola, Karinkanta, Kannus, \& Sievänen, 2015), while Caucasian and AA older adults who added aerobics training to balance and strength training did (Matsuda, Shumway-Cook, \& Ciol, 2010). Although exercise programs for DGI improvement involve balance and mobility interventions (Shumway-Cook et al., 1997), the specificity of exercise (walking) in the aerobics group - a major component of TUG and DGI - may explain their superior functional ability. Moreover, using muscle-strengthening and endurance exercises to improve FR performance (Cress et al., 1999) can explain the superior FR scores of the combination group over the aerobics group.

No differences in standing balance (sway) between exercise groups by type existed. Most participants exhibited low scores in sway indicating a low risk of falls (Table 6), and thus a potential reason for the lack of differences. In a study by Merom et al (2014), swimming with or without walking was linked to improved postural sway among older Caucasian men. Given that swimming is linked to strength parameters, especially among women (Garrido et al., 2012), the results by Merom et al (2014) reinforce this study's findings that both aerobic and strength training ac- 
Table 7. Differences in falls risks by exercise minutes

\begin{tabular}{|c|c|c|c|c|c|c|}
\hline \multicolumn{7}{|c|}{ Exercise minutes } \\
\hline Variable & Active $n=6$ & Somewhat active $n=6$ & $F^{* 1,9}$ & $\mathbf{p}$ & $\eta^{2}$ variance $(\%)$ & d Effect size \\
\hline \multicolumn{7}{|l|}{ DGI } \\
\hline Mean & 22.83 & 21.33 & 1.78 & 0.22 & 10.4 & $0.62 * *$ \\
\hline SD & 0.98 & 3.27 & & & & \\
\hline \multicolumn{7}{|c|}{ 6MWT (feet) } \\
\hline Mean & 1509 & 1370 & 1.17 & 0.31 & 7.1 & $0.50 * *$ \\
\hline SD & 318 & 225 & & & & \\
\hline \multicolumn{7}{|c|}{ TUG (seconds) } \\
\hline Mean & 10.57 & 10.40 & 0.02 & 0.89 & 0.10 & -0.06 \\
\hline SD & 3.27 & 2.50 & & & & \\
\hline \multicolumn{7}{|c|}{ FR (inches) } \\
\hline Mean & 14.17 & 13.58 & 0.08 & 0.79 & 0.76 & 0.16 \\
\hline SD & 3.86 & 3.44 & & & & \\
\hline \multicolumn{7}{|c|}{ Sway-EO (cm/s) } \\
\hline Mean & 1.72 & 1.38 & 1.77 & 0.22 & 7.6 & -0.52 \\
\hline SD & 0.81 & 0.43 & & & & \\
\hline \multicolumn{7}{|c|}{ Sway-EC (cm/s) } \\
\hline Mean & 1.91 & 1.67 & 0.64 & 0.45 & 4.3 & -0.37 \\
\hline SD & 0.69 & 0.60 & & & & \\
\hline
\end{tabular}

DGI: Dynamic gait index; 6MWT: Six-Minute walk test; TUG: Timed up and go; FR: Functional reach; EO: Eyes open; EC: Eyes closed $* F$ degrees of freedom for FR: 1,10 - ANOVA **Medium effect sizes for DGI, six-minute walk, and sway-EO

tivities are important to standing sway. For example, a combination of mainly aerobic training with some balance and strength training improved sway among older adults (Seco et al., 2012). The lack of differences in 6MWT between exercise groups by type were supported in intervention studies among post-stroke older adults (Mayo, MacKay-Lyons, Scott, Moriello, \& Brophy, 2013) and older healthy women (Wanderley et al., 2015). Aerobic training alone may provide benefits in functional capacity measured by 6MWT for older people, especially those who are not familiar with other types of exercises, such as resistance and balance training, or do not have the time or equipment to participate in multiple exercise modalities. Better scores for the active group in DGI and 6MWT over the somewhat active group reinforce the important role of continual training on falls risk prevention (Mayo et al., 2013). There were no differences in TUG and FR based on exercise minutes. Given the linkages of TUG and FR to aerobic and resistance training (Cress et al., 1999), it is possible that fewer minutes of aerobic exercise than those recommended for cardiovascular benefits can improve TUG and FR scores. Sondra and Elena were the only two active participants who were at increased falls risk on sway-EO, possibly explaining the lower sway in the somewhat active group than the active group. Sondra had multiple sclerosis (neuromuscular problems) and Elena had diabetes, conditions linked to increased risk of falls (Crews et al., 2013; Pieterse et al., 2006). Increased (worse) sway also corresponds to the presence of pain (Kanekar, Lee, \& Aruin, 2014) as observed for Elena and multiple sclerosis (Ruhe, Fejer, \& Walker, 2011) reported by Sondra.
According to the thematic analysis, most participants attributed their health problems to genetics and unhealthy diet. Although certain genes are linked to longevity among centenarians, the genetic factor seems to play a small role on healthy aging (Barzilai, Gabriely, Atzmon, \& Suh, 2010). Lifestyle choices such as proper diet and exercise, and environmental factors are the major contributors to healthy aging (Bernstein \& Munoz, 2012). Linking the qualitatively derived themes with the quantitative data (falls risks), regularly active women with an overall low falls risk (e.g., Margaret, Queran, and Rena) or women who were close to meeting exercise recommendations (Queran) reinforced the importance of exercise and proper diet to health. Being influenced by her own parents, Queran was able to reverse the negative health outcomes of perceived racial discrimination, reinforcing the resilience in the female AA culture (Airhihenbuwa \& Liburd, 2006). Although Elena and Edith linked aging to some of their health problems, they did not view aging as a barrier to their active lifestyles. Given that most women's risk of falls was linked to aging issues (e.g., increased risk in DGI - see Table 4), their positive views of aging may accompany their overall low risk of falls. Similarly, positive perceptions of aging associated more strongly with preventive health behaviors like exercise and diet than negative perceptions of aging (Levy \& Myers, 2004).

The second theme was falls prevention, encompassing bone and joint health, fitness, and functional independence. As also supported in the literature, musculoskeletal health (e.g., bone and joint health) and increased fitness and functioning are linked to falls prevention (Briggs, Cross, Hoy, 
Sànchez-Riera, Blyth, \& Woolf, 2016; Panel on Prevention of Falls in Older Persons, AGS/BGS, 2011). Nine women indicated the significance of exercise to prevent falls and six of those women exercised regularly. Some of those women were at increased risk of falls in mainly one fall-risk measure (DGI). The six active women and three somewhat active women acknowledged the importance of exercise on falls prevention possibly due to meeting or approaching the recommended exercise guidelines and/or being aware of falls risks associated with older age or neuromuscular problems like peripheral neuropathy (Crews et al., 2013; Pieterse et al., 2006). Therefore, even irregularly active people or those with a certain risk of falls can realize the key role of exercise on falls prevention not only via increased physical function, but also via the management of certain health conditions such as diabetes.

Although conservatively the sample size would be considered small for the quantitative data, it was conceptually and methodologically sufficient in this phronetic (pragmatic) research project (Flyvbjerg, 2001). While statistically the sample size was small for the omnibus $F$ tests, especially for the aerobics group, the use of effects sizes strengthened the value of the data. Although study participants developed health problems linked to inactivity and falls risks (e.g. high blood pressure, high cholesterol, diabetes, and neuromuscular disorders), their overall falls risk level was low, possibly because they were regularly active or somewhat active. Therefore, study generalizability is limited to cases similar to the studied population. This was not an experimental design and as such, identified differences do not necessarily reflect cause-and-effect relations. To our knowledge, this is the first phronetic mixed methods study to examine differences in falls risks by exercise type and exercise minutes as well as exercise values in relation to health, exercise behavior, falls prevention, and falls risks. To summarize how the qualitative data were linked to the quantitative data, three key trends were observed. First, active people with or without risk of falling realized the importance of exercise on falls risk prevention via improved physical function and health management. Although most women reported several health problems and the links between inactivity and cardiovascular disease among older adults are known in the literature (Lenz, Swartz, \& Strath, 2014), only one active participant with low falls risks linked inactivity to health issues. In general, active women with low falls risks reinforced the importance of exercise and diet on health. While aging was linked to increased falls risks (e.g., DGI), two older women approached aging positively and not detrimental to their functioning. The identified large and medium effect sizes reinforce the meaningfulness of the study findings and sample quality. Shedding new light on the controversy over the role of aerobic exercise in falls prevention, aerobic activities were linked to mobility-related falls risk tasks, such as dynamic balance (DGI) and mobility (TUG) for the studied sample. Participants reflected an understudied population, which was assessed within a comfortable environment in their community. The rich data set obtained from quantitative and qualitative assessments can assist practitioners with the development of exercise interventions. Implication for physical activity promoters (practitioners) working with at least somewhat active older AA women of low falls risks include: a) exercise programs for fall risk reduction should include not only muscle strengthening and balance activities, but also aerobic exercises; b) aerobic activities may be the preferred (only) exercise modality for older adults, especially those without the time for and/or knowledge about resistance or balance training; c) older adults should meet minimum exercise recommendations for the reduction of falls risks; d) beyond healthy diet, older people should understand that exercise participation can reduce the risk of falls via the prevention or management of certain health conditions (e.g. diabetes); e) although the role of genetics on health problems can be discussed, promoting healthy lifestyles and racial equity and downplaying the aging process may be more effective mechanisms for healthy aging; and e) the importance of exercise on falls risk reduction needs to be communicated among inactive or irregularly active older adults, who may not be familiar with this recommendation. Suggestions for future research in older AA women involve: a) conducting experimental designs to examine differences in falls risks based on exercise type (e.g. endurance training vs. combined programs of endurance, strength training, and balance activities) and exercise adherence; b) examining differences in lifestyle values via linkages to health, falls risks, and fear of falling based on gender, race, and setting (e.g. urban vs. rural); c) investigating differences in health and healthy lifestyle values based on exercise levels (e.g. active vs. inactive); and d) developing exercise promotion programs based on community members' input regarding their values about health and healthy lifestyles: is it only my bad genes that contribute to my health problems or is it also my unhealthy diet and inactivity?

\section{CONCLUSION}

To our knowledge, this is the first mixed methods phronetic study to examine the relations among exercise behavior and values, health, and falls risks among community-dwelling older AA women, an understudied population. Based on the study findings, exercise promoters should emphasize not only muscle strengthening and balance activities, but also aerobic training for falls prevention. Meeting minimum exercise recommendations is also key to falls risk reduction. Understanding the importance of exercise on health and falls prevention is crucial among diverse older AA women, especially those of lower exercise levels.

\section{REFERENCES}

Airhihenbuwa, C. O., \& Liburd, L. (2006). Eliminating health disparities in the African American population: The interface of culture, gender, and power. Health Education \& Behavior, 33, 488-501. doi: 10.1177/1090198106287731

Ambrose, A. F., Paul, G., \& Hausdorff, J. M. (2013). Risk factors for falls among older adults: A review of the 
literature. Maturitas, 75, 51-61. doi:10.1016/j.maturitas.2013.02.009

Aristotle. (1962). Nichomachean ethics (M. Ostwald, Trans.). Indianapolis, IN: Bobbs-Merrill. (Original work published 350 B.C.E).

Barzilai, N., Gabriely, I., Atzmon, G., Suh, Y., Rothenberg, D., \& Bergman, A. (2010). Genetic studies reveal the role of the endocrine and metabolic systems in aging. The Journal of Clinical Endocrinology \& Metabolism, 95, 4493-4500. doi:10.1210/jc.2010-0859

Bernstein, M., \& Munoz, N. (2012). Position of the Academy of Nutrition and Dietetics: Food and nutrition for older adults: Promoting health and wellness. Journal of the Academy of Nutrition and Dietetics, 112, 1255-1277. doi: 10.1016/j.jand.2012.06.015

Briggs, A. M., Cross, M. J., Hoy, D. G., Sànchez-Riera, L., Blyth, F. M., \& Woolf, A. D. (2016). Musculoskeletal health conditions represent a global threat to healthy aging: A report for the 2015 World Health Organization World Report on Ageing and Health. Gerontologist, 56, S243-S255. doi:https://oi.org/10.1093/geront/gnw002

Canadian Society for Exercise Physiology. (1994). PAR-Q \& you. Gloucester, Ontario: Author.

Cohen, J. (1988). Statistical power analysis for the behavioral sciences ( $2^{\text {nd }}$ Ed.). Hillsdale, NJ: Erlbaum.

Cortina, J. M., \& Nouri, H. (2000). Effect size for ANOVA designs. Thousand Oaks, CA: Sage.

Cress, E. M., Buchner, D. M., Questad, K. A., Esselman, P. C., deLateur B. J., \& Schwartz, R. S. (1999). Exercise effects on physical functional performance in independent older adults. Journal of Gerontology: Medical Sciences, S4A, M242-M248.

Crews, R. T., Yalla, S. V., Fleischer, A. E., \& Wu, S. C. (2013). A growing troubling triad: Diabetes, aging, and falls. Journal of Aging Research, 2013, 1-6. doi: $10.1155 / 2013 / 342650$

Duncan, P. W., Studenski, S., Chandler, J., \& Prescott, B. (1992). Functional reach: Predictive validity in a sample of elderly male veterans. Journal of Gerontology, 47, M93-98.

Enright, P. L. (2003). The six-minute walk test. Respiratory Care, 48, 783-785.

Federal Interagency Forum on Aging-Related Statistics. Older Americans 2016: Key Indicators of Well-Being. Retrieved from http://www.agingstats.gov/docs/LatestReport/OA2016.pdf

Flyvbjerg, B. (2001). Making social science matter: Why social inquiry fails and how it can succeed again. Cambridge, England: Cambridge University Press.

Flyvbjerg,B. (2004). Phronetic planning research: Theoretical and methodological reflections. Planning Theory \& Practice, 5, 283-306. doi:10.1080/1464935042000250195

Folstein, M. F., Folstein, S. E., \& McHugh, P. R. (1975). "Mini-mental state": A practical method for grading the cognitive status of patients for the clinician. Journal of Psychiatric Research, 12, 189-198.

Franco, M. R., Tong, A., Howard, K., Sherrington, C., Ferreira, P. H., Pinto, R. Z., \& Ferreira, M. L. (2015). Older people's perspectives on participation in physical activi- ty: A systematic review and thematic synthesis of qualitative literature. British Journal of Sports Medicine, 49, 1268-1276. doi:10.1136/bjsports-2014-094015

Garrido, N. D., Silva, A. J., Fernandes, R. J., Barbosa, T. M., Costa, A. M., Marinho, D., \& Marques, M. C. (2012). High level swimming performance and its relation to non-specific parameters: A cross-sectional study on maximum handgrip isometric strength. Perceptual and Motor Skills, 114, 936 - 948. doi:10.2466/05.10.25. PMS.114.3.936-948

Haynes-Maslow, L., Parsons, S. E., Wheeler, S. B., \& Leone, L. A. (2013). A Qualitative Study of perceived barriers to fruit and vegetable consumption among low-income populations. Preventing Chronic Disease, 10, 1-10. doi: http://dx.doi.org/10.5888/pcd10.120206

Inouye, S. K., Brown, C. J., \& Tinetti, M. E. (2009). Medicare nonpayment, hospital falls, and unintended consequences. The New England Journal of Medicine, 360, 2390-2393.

Kafle, N. P. (2011). Hermeneutic phenomenological research method simplified. Bodhi: An Interdisciplinary Journal, 5, 181-200. doi: http://dx.doi.org/10.3126/bodhi. v5i1.8053

Kanekar, N., Lee, Y. J., \& Aruin, A. S. (2014). Frequency analysis approach to study balance control in individuals with multiple sclerosis. The Journal of Neuroscience Methods, 222, 91-96. doi: 10.1016/j.jneumeth.2013.10.020

Karlsson, M. K., Vonschewelov, T., Karlsson, C., Cöster, M., \& Rosengen, B. E. (2013). Prevention of falls in the elderly: A review. Scandinavian Journal of Public Health, 41, 442-454. doi: 10.1177/1403494813483215

Kline, R. B. (2015). Principles and practice of structural equation modeling ( $4^{\text {th }}$ ed.). New York, NY: Guilford Press.

Kosma, M., Buchanan, D. R., \& Hondzinski, J. (2015). The role of values in promoting physical activity. Quest, 67, 241-254. doi:10.1080/00336297.2015.1050117

Kosma, M., Buchanan, D. R., \& Hondzinski, J. (in press). Complexity of exercise behavior among older African American women. Journal of Aging and Physical Activity.

Kosma, M., Cardinal, B.J., \& McCubbin, J.A. (2004). Recruitment techniques among understudied populations and their implications for physical activity promotion. Quest, 56, 413-420.

Lakens, D. (2013). Calculating and reporting effect sizes to facilitate cumulative science: A practical primer for t-tests and ANOVAs. Frontiers in Psychology, 4, 863. doi:10.3389/fpsyg.2013.00863

Lenz, E. K., Swartz, A. M., \& Strath, S. J. (2014). Do sedentary behaviors modify the health status of older adults? International Journal of Kinesiology \& Sports Science, 2, 13-22. doi:10.7575/aiac.ijkss.v.2n.1p.13

Levine, T. R., \& Hullett, C. R. (2002). Eta squared, partial eta squared, and misreporting of effect size in communication research. Human Communication Research, 28, 612-625. doi:10.1111/j.1468-2958.2002.tb00828.x

Levy, B. R., \& Myers, L. M. (2004). Preventive health behaviors influenced by self-perceptions of aging. 
Preventive Medicine, 39, 625-629. doi: 10.1016/j. ypmed.2004.02.029

Liu-Ambrose, T. Y. L., Khan, K. M., Eng, J. J., Gillies, G. L., Lord, S. R., \& McKay, H. A. (2005). The beneficial effects of group-based exercises on fall risk profile and physical activity persist 1 year postintervention in older women with low bone mass: Follow-up after withdrawal of exercise. Journal of the American Geriatrics Society, 53, 1767-1773. Doi:10.1111/j.1532-5415.2005.53525.x

Matsuda, P. N., Shumway-Cook, A., \& Ciol, M. A. (2010). The effects of a home-based exercise program on physical function in frail older adults. The Journal of Geriatric Physical Therapy, 33, 78-84.

Mayo, N. E., MacKay-Lyons, M. J., Scott, S. C., Moriello, C., \& Brophy, J. (2013). A randomized trial of two homebased exercise programmes to improve functional walking post-stroke. Clinical Rehabilitation, 27, 659-671. doi: $10.1177 / 0269215513476312$

Melzer, I., Benjuya, N., \& Kaplanski, J. (2004). Postural stability in the elderly: A comparison between fallers and non-fallers. Age and Ageing, 33, 602-607. doi: https:// doi.org/10.1093/ageing/afh218

Merom, D., Stanaway, F. F., Handelsman, D. J., Waite, L. M., Seibel, M. J., Blyth, F. M.,... Cumming, R. G. (2014). Swimming and other sporting activities and the rate of falls in older men: Longitudinal findings from the Concord Health and Ageing in Men Project. American Journal of Epidemiology, 180, 830-837. doi: https://doi. org/10.1093/aje/kwu199.

Moghadam, M., Ashayeri, H., Salavati, M., Sarafzadeh, J., Taghipoor, K. D., Saeedi, A., \& Salehi, R. (2011). Reliability of center of pressure measures of postural stability in healthy older adults: Effects of postural task difficulty and cognitive load. Gait \& Posture, 33, 651-655. doi: $10.1016 /$ j.gaitpost.2011.02.016

Panel on Prevention of Falls in Older Persons, American Geriatrics Society and British Geriatrics Society. (2011). Summary of the updated American Geriatrics Society/ British Geriatrics Society clinical practice guideline for prevention of falls in older persons. The Journal of the American Geriatrics Society, 59, 148-157.

Paterson, D. H., \& Warburton, D. E. (2010). Physical activity and functional limitations in older adults: A systematic review related to Canada's Physical Activity Guidelines. International Journal of Behavioral Nutrition and Physical Activity, 7, 38. doi: 10.1186/1479-5868-7-38

Patil, R., Uusi-Rasi, K., Tokola, K., Karinkanta, S., Kannus, P., \& Sievänen, H. (2015). Effects of a multimodal exercise program on physical function, falls, and injuries in older women: A 2-year community-based, randomized controlled trial. Journal of the American Geriatrics Society, 63, 1306-1313. doi: 10.1111/jgs.13489

Pieterse, A. J., Luttikhold, T. B., de Laat, K., Bloem, B. R., van Engelen, B. G., \& Munneke, M. (2006). Falls in patients with neuromuscular disorders. The Journal of the Neurological Sciences, 21, 87-90. doi: 10.1016/j.jns.2006.09.008

Podsiadlo, D., \& Richardson, S. (1991). The timed "Up \& Go": A test of basic functional mobility for frail elderly persons. Journal of the American Geriatrics Society, 39, 142-148

Ruhe, A., Fejer, R., \& Walker, B. (2011). Center of pressure excursion as a measure of balance performance in patients with non-specific low back pain compared to healthy controls: A systematic review of the literature. European Spine Journal, 20, 358-368. doi: 10.1007/ s00586-010-1543-2

Sadeghi, H., Hakim, M. N., Hamid, T. A., Amri, S. B., Razeghi, M., Farazdaghi, M., \& Shakoor, E. (2017). The effect of exergaming on knee proprioception in older men: A randomized controlled trial. Archives of Gerontology \& Geriatrics, 69, 144-150. doi:10.1016/j. archger.2016.11.009

Seco, J., Abecia, L. C., Echevarria, E., Barbero, I., Torres-Unda, J., Rodriguez, V., \& Calvo, J. I. (2012). A long-term physical activity training program increases strength and flexibility, and improves balance in older adults. Rehabilitation Nursing, 38, 37-47. doi: 10.1002/ rnj.64

Sherrington, C., Tiedemann, A., Fairhall, N., Close, J. C. T., \& Lord, S. R. (2011), Exercise to prevent falls in older adults: An updated meta-analysis and best practice recommendations. The NSW Public Health Bulletin, 22, 78-83. doi: 10.1071/NB10056

Shumway-Cook, A., Baldwin, M., Polissar, N. L., \& Gruber, W. (1997). Predicting the probability for falls in community-dwelling older adults. Physical Therapy, $77,812-819$

Shumway-Cook, A., Gruber, W., Baldwin, M., \& Liao, S. (1997). The effect of multidimensional exercises on balance, mobility, and fall risk in community-dwelling older adults. Physical Therapy, 77, 46-57.

Sigal, R. J., Kenny, G. P., Wasserman, D. H., \& Castaneda-Sceppa, C. (2004). Physical activity/exercise and type 2 diabetes. Diabetes Care, 27, 2518-2539.

Steffen, T. M., Hacker, T. A., \& Mollinger, L. (2002). Age- and gender-related test performance in community-dwelling elderly people: Six-Minute Walk Test, Berg Balance Scale, Timed Up \& Go Test, and gait speeds. Physical Therapy, 82, 128-137.

Thapa, P. B., Gideon, P., Brockman, K. G., Fought, R. L., \& Ray, W. A. (1996). Clinical and biomechanical measures of balance as fall predictors in ambulatory nursing home residents. Journal of Gerontology: Medical Sciences, 51, M239-246.

U.S. Department of Health and Human Services, Office of Disease Prevention and Health Promotion. (2016a). Healthy People 2020: Topics and Objectives-Physical Activity. Retrieved from http://www.healthypeople. gov/2020/topics-objectives/topic/physical-activity

U.S. Department of Health and Human Services, Office of Disease Prevention and Health Promotion. (2016b). Healthy People 2020: Topics and Objectives-Diabetes. Retrieved from https://www.healthypeople.gov/2020/ data-search/Search-the-Data\#topic-area $=3514$

U.S. Department of Health and Human Services, Office of Disease Prevention and Health Promotion. (2016c). Healthy People 2020: Topics and Objectives-Heart 
Disease and Stroke. Retrieved from https://www.healthypeople.gov/2020/data-search/Search-the-Data\#topicarea $=3516$

Wanderley, F. A. C., Oliveira, N. L., Marques, E., Moreira, P., Oliveira, J., \& Carvalho, J. (2015). Aerobic versus resistance training effects on health-related quality of life, body composition, and function of older adults. Journal of Applied Gerontology, 34, NP143-165. doi: 10.1177/0733464812468502

Wolf, S. (2015). The variety of values: Essays on morality, meaning, \& love. New York, NY: Oxford University Press. 